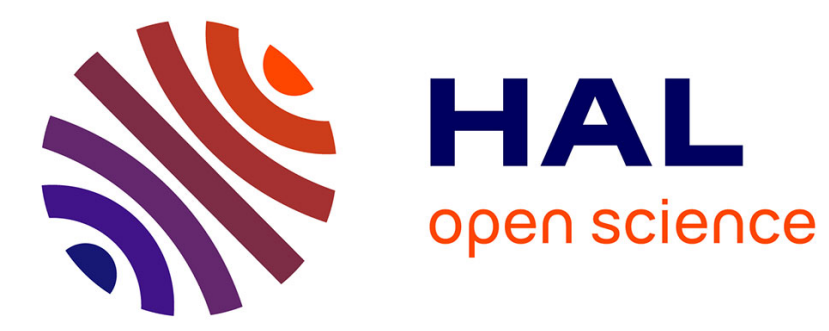

\title{
Robust Microscale Grasping Through a Multimodel Design : Synthesis and Real Time Implementation.
}

\author{
Mokrane Boudaoud, Marcelo Gaudenzi de Faria, Yassine Haddab, Sinan
}

Haliyo, Yann Le Gorrec, Philippe Lutz, Stéphane Régnier

\section{To cite this version:}

Mokrane Boudaoud, Marcelo Gaudenzi de Faria, Yassine Haddab, Sinan Haliyo, Yann Le Gorrec, et al.. Robust Microscale Grasping Through a Multimodel Design: Synthesis and Real Time Implementation.. Control Engineering Practice, 2015, 39, pp.12-22. 10.1016/j.conengprac.2014.11.003 . hal-02868181

\section{HAL Id: hal-02868181 \\ https://hal.science/hal-02868181}

Submitted on 15 Jun 2020

HAL is a multi-disciplinary open access archive for the deposit and dissemination of scientific research documents, whether they are published or not. The documents may come from teaching and research institutions in France or abroad, or from public or private research centers.
L'archive ouverte pluridisciplinaire HAL, est destinée au dépôt et à la diffusion de documents scientifiques de niveau recherche, publiés ou non, émanant des établissements d'enseignement et de recherche français ou étrangers, des laboratoires publics ou privés. 


\title{
Robust Microscale Grasping Through a Multimodel Design: Synthesis and Real Time Implementation
}

\author{
Mokrane Boudaoud* Marcelo Gaudenzi De Faria** \\ Yassine Haddab ${ }^{* *}$ Sinan Haliyo* Yann Le Gorrec ${ }^{* *}$ \\ Philippe Lutz** Stéphane Régnier* \\ * Institut des Systèmes Intelligents et de Robotique, Université Pierre et Marie \\ Curie, CNRS UMR 7222, 4 Place Jussieu, F-75252 Paris Cedex, France \\ ** FEMTO-ST Institute, UMR CNRS 6174 - UFC-ENSMM-UTBM; Automatic \\ Control and Micro-Mechatronic Systems (AS2M) Department; 24, rue Alain \\ Savary, 25000 Besançon - France
}

\begin{abstract}
:
This paper deals with robust force control at the microscale for safe manipulation of deformable soft materials. Since mechanical properties of micrometer sized objects are highly uncertain, instability often occurs during a gripping task. This leads to object damage or destruction due to excessive gripping force. In this paper we propose the design of a robust dynamic output feedback controller able to insure desired performances for a set of 65 soft and resilient microspheres whose diameter ranges from $40 \mu \mathrm{m}$ to $80 \mu \mathrm{m}$ and stiffness varies from $2.8 \mathrm{~N} / \mathrm{m}$ to $15.7 \mathrm{~N} / \mathrm{m}$. The degrees of freedom of the controller are managed by the use of a set of elementary observers. Robustness with respect to parametric uncertainties is satisfied thanks to an iterative procedure alternating between multimodel closed loop eigenstructure assignment and worst case analysis. The developed controller is of low order and can be implemented in real time. Robust gripping force control is for the first time demonstrated experimentally when dealing with the manipulation of a large number of variable deformable soft materials at the microscale. Both simulations and experimental results validate the interest of such control design approach.
\end{abstract}

Keywords: Microelectromechanical systems, Force feedback, Robust control, Eigenstructure Assignment, Grasping.

\section{INTRODUCTION}

Microrobotics holds promises for the efficient and safe manipulation of biologic samples and living cells (Liu et al. [2009]). In precursor works, techniques inspired from Atomic Force Microscopy have proven a reliable tool for the characterization of biomaterials. Muller et al. [2009] used an AFM probe to obtain high resolution force images of living cells. Boukallel et al. [2009] proposed an improved probe design to measure mechanical characteristics of cells in long traction/compression cycles. Desmaele et al. [2012] introduced a dynamic measurement method improving the overall reliability. However, single cantilevers or similar designs are is ill-adapted for manipulation. Recently, the development of dedicated microgrippers that include both actuation and force sensing in dimensions adapted to biologic samples opened the way to novel and cost-effective applications ranging from in-vitro fertilization to genetics (Carrozza et al. [2000]; Beyeler et al. [2007]).

Biologic cells are highly deformable soft materials. They are very sensitive to applied force and to how they are handled. Consequently, the use of grippers for their manipulation calls for a precise force control of grasping. This particular issue is evidently not limited to biologic samples and is a general concern for micro and nanoscale manipulation. To apply safe gripping forces required for

\footnotetext{
$\star$-.Control

Engineering

Practice.

E-mail:

mokrane.boudaoud@isir.upmc.fr; marcelo.gaudenzi@femto-st.fr yassine.haddab@femto-st.fr; sinan.haliyo@upmc.fr; legorrec@femtost.fr; philippe.lutz@femto-st.fr; stephane.regnier@upmc.fr.
}

the manipulation of soft objects, several solutions have been reported. Bolopion et al. [2012] use haptic feedback allowing the user to interact with microscale objects and leaves the force control to the operator. In a more traditional approach, feedback control (Carrozza et al. [2000]; Park et al. [2005]; Liu et al. [2009]) allows for an automated approach to gripping.

At the microscale, soft materials have mechanical properties, namely stiffness and damping, close to that of the actuation and sensing systems of microgrippers. Therefore, during gripping tasks, samples have enough variation to induce instabilities that damage the gripper or the sample. In the literature, microscale force feedback control designs are often based on PI, PID or LQG schemes (Carrozza et al. [2000]; Park et al. [2005]; Liu et al. [2009]; Boudaoud et al. [2013]). Controller synthesis is often achieved considering the mechanical properties of a single sample and closed loop performance are validated experimentally when gripping the sample used for the synthesis (Liu et al. [2009]; Boudaoud et al. [2013]). These approaches lack the robustness required for micromanipulation. To overcome this problem, $\mathrm{H}_{\infty}$ controllers such as proposed in Rakotondrabe et al. [2007]; Rakotondrabe et Le Gorrec. [2010] are often used. Resulting schemes allow for a robust force control, but such controllers are often of high order and can be difficult to implement in real time due to the considered bandwidth (Poussot-Vassal et al. [2008]; Boudaoud et al. [2012]).

The aim of the present work is to propose a robust control design procedure aiming at deriving efficient low 
order controllers. The synthesis is based on a closed loop eigenstructure assignment methodology as previously mentioned in Boudaoud et al. [2012]. The shortcoming of this approach is that it can not deal with uncertain and unmeasurable parameters. In gripping tasks, the size, the stiffness and the damping of the samples are part of uncertain parameters. To overcome this issue, based on a set of elementary observers, multi-model assignment constraints are defined using an iterative procedure (Magni et al. [1998]). The most relevant multimodel constraints are defined considering parametric uncertainties in a set of 65 soft and resilient microspheres with diameters ranging from $40 \mu \mathrm{m}$ to $80 \mu \mathrm{m}$ and stiffness from $2.8 \mathrm{~N} / \mathrm{m}$ to $15.7 \mathrm{~N} / \mathrm{m}$. The order of the controller is equal to the number of observers, and is potentially low.

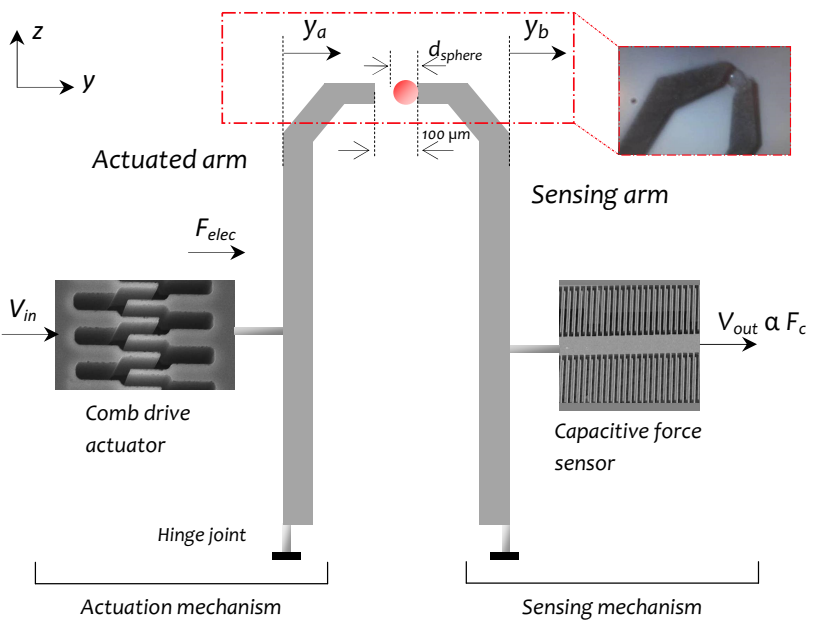

Fig. 1. Simplified scheme of the microgripper.

The proposed approach is designed considering an electrostatic microgripper with an integrated force sensor and the set of microspheres with varying size and stiffness. For control purposes, a non-linear coupled model of the microgripper with an object is established. The non-linear model is formulated in Section 3 into an uncertain Linear Time Invariant (LTI) model taking into account the dynamics of the actuator, the force sensor and the gripped microsphere. Gripping force control is then achieved in Section 4 through the above mentioned methodology. Results presented in Section 6 show the first experimental demonstration of robust gripping force control at the microscale for the manipulation of a large number of soft materials with varying mechanical properties. In Section 7 , the applicable range of the control design for microscale objects grasping is discussed.

\section{SPECIFICATIONS OF THE MICROGRIPPER AND SAMPLE MICROSPHERES}

The microgripper used in this study is Fem [2009]. The first version of this microgripper was described in Beyeler et al. [2007]. A detailed description of its architecture and working principle can be found in Boudaoud et al. [2013]. This two fingers (arms) microgripper depicted in Fig. 1 includes comb-drive actuation on one part and capacitive force sensing on the other. The gap of the gripper is $100 \mu \mathrm{m}$.

Manipulated samples are thermoplastic particles called expancel microspheres. Expancel are thermoplastic microspheres enclosing hydrocarbon. These microspheres expand when heated, producing many applications. They are deformable, soft and resilient, with properties close to that of biological samples (M. Kemper. [2004]) which makes them very attractive for force control experiments at the microscale. Three kinds of microspheres have been used in this study: model 1, model 2 and model 3 (Table 1). For each model, the manufacturer provides the size, with uncertainties of about $10 \%$ as shown in Table 1 , but the stiffness and the damping are not known. Hence, the size and both the stiffness and the damping of a set of 65 microspheres are experimentally identified in section 3.2 using the microgripper.

Table 1. Reference, diameter and number of characterized Expancel microspheres.

\begin{tabular}{llll}
\hline Model & Reference & Diameter & $\begin{array}{l}\text { Number } \\
\text { samples }\end{array}$ \\
\hline Model 1 & Akz1 [2011] & $57 \mu \mathrm{m} \pm 14 \mu \mathrm{m}$ & 25 \\
\hline Model 2 & Akz2 [2011] & $55 \mu \mathrm{m} \pm 5 \mu \mathrm{m}$ & 17 \\
\hline Model 3 & Akz3 [2011] & $68 \mu \mathrm{m} \pm 10 \mu \mathrm{m}$ & 23 \\
\hline
\end{tabular}

\section{NON-LINEAR MODELING OF THE GRASPING}

\subsection{Modeling of the gripper}

In Boudaoud et al. [2012], a non-linear model of the actuation mechanism (Fig. 1) is described along with an experimental validation. It is a mass-spring-damper model where the stiffness $\left(k_{a}\right)$ and the damping $\left(d_{a}\right)$ are nonlinear polynomial functions of the position of the actuated arm $y_{a}$ :

$$
\begin{aligned}
& k_{a}\left(y_{a}\right)=\sum_{i=1}^{6} k_{i a} y_{a}^{i-1} \\
& d_{a}\left(y_{a}\right)=\sum_{i=1}^{4} d_{i a} y_{a}^{i}
\end{aligned}
$$

$k_{i a}$ and $d_{i a}$ are constant coefficients of the stiffness and the damping polynomial functions respectively.

This mass-spring-damper model is extended to the case where a microsphere is gripped between the actuated and sensing arms. The model of the overall system is obtained by coupling both the non-linear model of the actuation mechanism with a linear mass-spring-damper model of the sensing mechanism and considering the gripped object as a spring-damper with a stiffness $k_{o}$ and a damping $d_{o}$. It leads to the following model:

$$
\begin{aligned}
{\left[\begin{array}{l}
\dot{y}_{a} \\
\ddot{y}_{a} \\
\dot{y}_{b} \\
\ddot{y}_{b}
\end{array}\right]=} & {\left[\begin{array}{cccc}
0 & 1 & 0 & 0 \\
-\frac{k_{o}+k_{a}\left(y_{a}\right)}{m_{a}} & -\frac{d_{o}+d_{a}\left(y_{a}\right)}{m_{a}} & \frac{k_{o}}{m_{a}} & \frac{d_{o}}{m_{a}} \\
0 & 0 & 0 & 1 \\
\frac{k_{o}}{m_{b}} & \frac{d_{o}}{m_{b}} & -\frac{k_{o}+k_{b}}{m_{b}} & -\frac{d_{o}+d_{b}}{m_{b}}
\end{array}\right]\left[\begin{array}{l}
y_{a} \\
\dot{y}_{a} \\
y_{b} \\
\dot{y}_{b}
\end{array}\right] } \\
+ & {\left[\begin{array}{l}
0 \\
\frac{N_{a} \varepsilon h_{z}}{2 m_{a} g D_{a}} \\
0 \\
0
\end{array}\right] V_{i n}^{2} } \\
F_{c} & =\left[\begin{array}{lll}
k_{o} & d_{o}-k_{o}-d_{o}
\end{array}\right]\left[\begin{array}{l}
y_{a} \\
\dot{y}_{a} \\
y_{b} \\
\dot{y}_{b}
\end{array}\right]
\end{aligned}
$$

where $y_{i}, k_{i}, d_{i}$ and $m_{i}$ are respectively the position, the stiffness, the damping and the mass of the actuated arm (when replacing $i$ with $a$ ) and the sensing arm (when replacing $i$ with $b$ ) as depicted in Fig. 1. $N_{a}, \varepsilon, h_{z}$, and $g$ 
are intrinsic parameters of the comb drive, providing the electrostatic force $F_{\text {elec }}\left(V_{i n}\right) . F_{c}$ is the gripping force, as measured through the capacitive sensor. $D_{a}$ is an amplification parameter. Numerical values of the model parameters are given in Boudaoud et al. [2012] and Boudaoud et al. [2013].
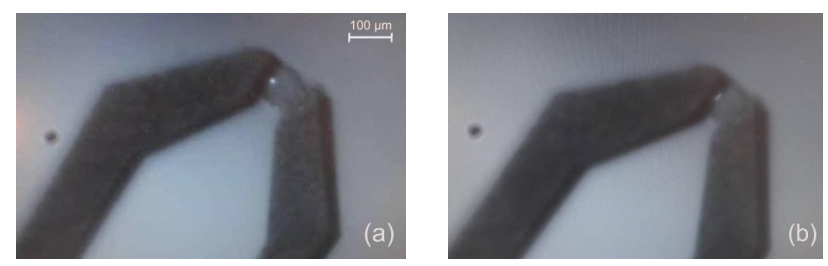

Fig. 2. The microgripper handling an expancel microsphere: uncompressed (a) and compressed (b).

\subsection{Experimental characterization of the microspheres}

In a random gripping case, the stiffness $\left(k_{o}\right)$, the damping $\left(d_{o}\right)$ and the diameter $\left(d_{\text {sphere }}\right)$ (Fig. 1$)$ of the gripped object are unknown. A robust control strategy would ideally be able to deal with these uncertainties. For the synthesis of such a controller, a set of 65 Expancel microspheres with varying $k_{o}, d_{o}$ and $d_{\text {sphere }}$ are experimentally identified. This dataset is then used, first for synthesis and then for validation.

At the microscale, three classes of adhesive forces are dominant (Chaillet et al. [2010]) :van der Waals forces, Coulomb forces and capillary forces. Adhesive forces often appear between the object and the gripping arms or between the object and the surface (substrate). In this paper, the identification of the microspheres and the gripping force control are performed when the object is compressed. The effect of adhesive forces are neglected.

The set of microspheres are characterized using solely the microgripper mounted on a 3 DoF stage. To apply a controlled gripping force on an object, the gripping arms must be in contact with the object. This preloading also avoid the dead zone in force control.

The user manually positions the gripper on the sphere until it contacts the sensing arm, as depicted in Fig. 1. In order to contact both arms on the object, the actuated arm of the gripper is fed with an input voltage $V_{i n 0}$ until the sensor detects a signal (i.e. the sensing arm starts moving). At this stage, the gripping force is lower than $2.35 \mu \mathrm{N}$ (measurement noise). The final position $y_{a}$ of the actuated arm is measured with an external laser interferometer (SP120 SIOS Meßtechnik GmbH). The $d_{\text {sphere }}$ is deduced from this measurement $\left(d_{\text {sphere }}=100 \mu \mathrm{m}-y_{a}\right)$.

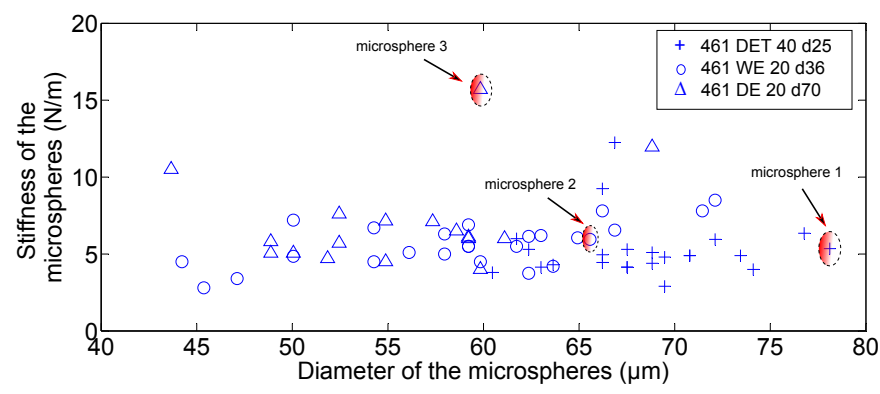

Fig. 3. Disparity map of diameter and stiffness of characterized Expancel microspheres.

A $10 \mathrm{~V}$ step signal is thereafter applied to the actuator, which compresses the grasped sample (Fig. 2). The step response provided by the force sensor is then used for the
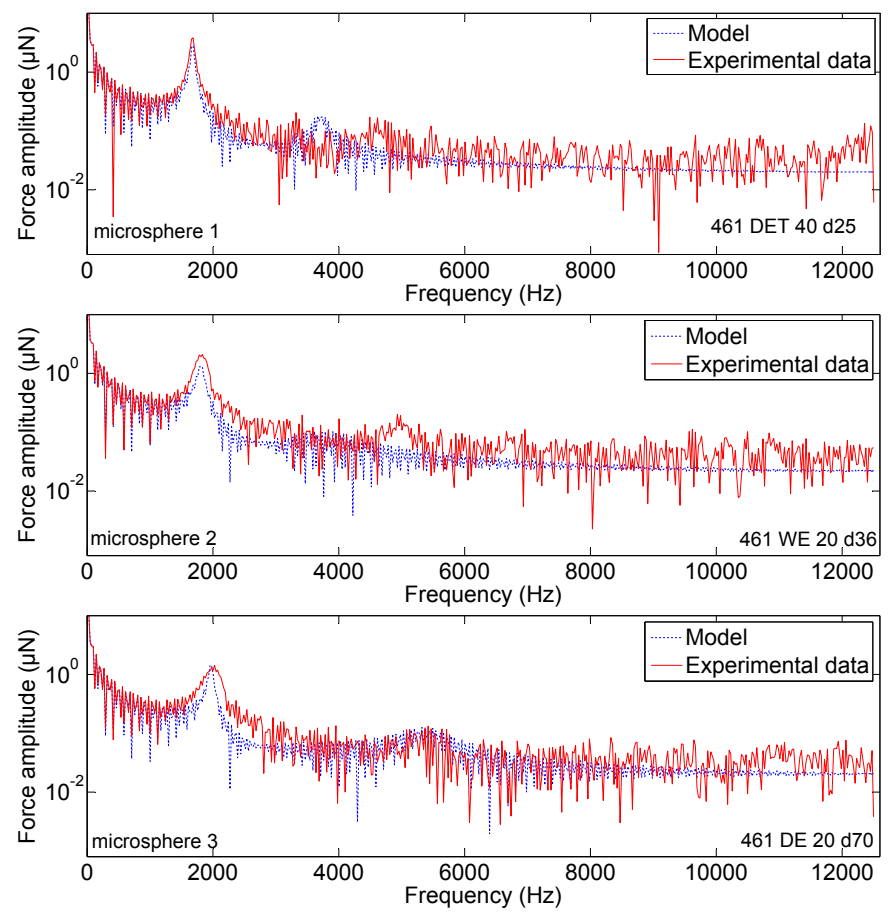

Fig. 4. Fast Fourier Transform of the gripping force signal for three different microspheres. The input signal is a $10 \mathrm{~V}$ step voltage.

identification of the stiffness $\left(k_{0}\right)$ and the damping $\left(d_{0}\right)$ using the non-linear coupled model given in eq. (3).

These parameters are identified using the Matlab software. The stiffness $k_{o}$ is identified such that the first resonance frequency of the system and that of the model are equal. The damping $d_{o}$ has been also identified from the experimental damping of the first resonance frequency. Hence, the second resonance frequency of the model does not match precisely with experimental one.

Size and stiffness identification results are shown in Fig. 3. $d_{\text {sphere }}$ ranges from $43.66 \mu \mathrm{m}$ to $78.11 \mu \mathrm{m}$ and $k_{o}$ varies between $2.8 \mathrm{~N} / \mathrm{m}$ and $15.7 \mathrm{~N} / \mathrm{m}$, the damping $d_{o}$ from $1.5 \times 10^{-6} \mathrm{Ns} / \mathrm{m}$ to $1.8 \times 10^{-4} \mathrm{Ns} / \mathrm{m}$. The first eigenmode of the coupled system is between $1.6 \mathrm{kHz}$ and $2 \mathrm{kHz}$. The uncertainty of the identification of the first resonance frequency of the grasping model is lower than $2.4 \%$. The mean static error between the model and experimental data for the set of the characterized spheres is $27.34 \%$.

Fig. 4 depicts the FFT (Fast Fourier Transform) of the output signal of the non-linear model (3) for three different microspheres from Table 1 . The input signal is a step of $10 \mathrm{~V}$ in amplitude. Simulation results are compared with experiments. Used microspheres are also shown on Fig. 3. The three spheres are part of the 65 characterized spheres. Experimental measurements have been obtained using the capacitive force sensor. Measurements have been performed with a $25 \mathrm{kHz}$ sampling frequency during $1 \mathrm{~s}$ (25000 samples).

\subsection{Linearization}

The controller will be designed considering the nonlinear model $G_{N L}$ (see Fig. 5) describing the dynamic transfer between the input $U$ and the output $F_{c}$, where $U=V_{i n}^{2}$. $G_{N L}$ is not the complete model of the real system, it is a part of it. As such, for the implementation of the controller, the square root of the control low $U$ will be applied on the 


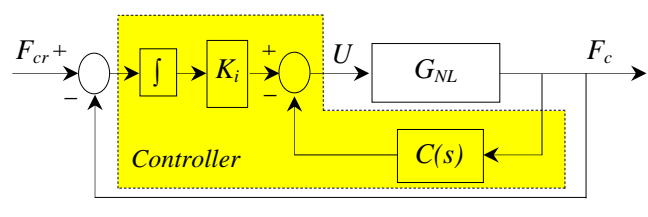

Fig. 5. Simplified scheme of the controller (dashed block) and the nonlinear model $G_{N L}$.

real system. This consideration is made because the control low $U$ must be always positive.

From eq. (3), $G_{N L}$ is given as follows:

$$
\begin{aligned}
{\left[\begin{array}{l}
\dot{y}_{a} \\
\ddot{y}_{a} \\
\dot{y}_{b} \\
\ddot{y}_{b}
\end{array}\right]=} & {\left[\begin{array}{cccc}
0 & 1 & 0 & 0 \\
-\frac{k_{o}+k_{a}\left(y_{a}\right)}{m_{a}} & -\frac{d_{o}+d_{a}\left(y_{a}\right)}{m_{a}} & \frac{k_{o}}{m_{a}} & \frac{d_{o}}{m_{a}} \\
0 & 0 & 0 & 1 \\
\frac{k_{o}}{m_{b}} & \frac{d_{o}}{m_{b}} & -\frac{k_{o}+k_{b}}{m_{b}} & -\frac{d_{o}+d_{b}}{m_{b}}
\end{array}\right]\left[\begin{array}{l}
y_{a} \\
\dot{y}_{a} \\
y_{b} \\
\dot{y}_{b}
\end{array}\right] } \\
+ & {\left[\begin{array}{l}
0 \\
\frac{N_{a} \varepsilon h_{z}}{2 m_{a} g D_{a}} \\
0 \\
0
\end{array}\right] U } \\
F_{c} & =\left[\begin{array}{lll}
k_{o} & d_{o}-k_{o}-d_{o}
\end{array}\right]\left[\begin{array}{l}
y_{a} \\
\dot{y}_{a} \\
y_{b} \\
\dot{y}_{b}
\end{array}\right]
\end{aligned}
$$

For each object in the dataset, the non-linear model $G_{N L}$ of eq. (4) is linearized in the neighborhood of the operating point $\delta_{a}$ of the actuated arm. For the comb drive actuator, the non-linear parameters $k_{a}\left(\delta_{a}\right)$ and $d_{a}\left(\delta_{a}\right)$ are polynomial. The governing parameter $\delta_{a}$ is the position of the actuated arm. Using a Jacobian linearization, the nonlinear plant of eq. (4) is formulated into an uncertain LTI model:

$$
\begin{aligned}
& G_{c}\left(\Delta_{c}\right)\left\{\begin{array}{l}
\dot{X}_{c}=A_{c}\left(\Delta_{c}\right) X_{c}+B_{c} U \\
F_{c}=C_{c}\left(\Delta_{c}^{\prime}\right) X_{c}
\end{array}\right. \\
& A_{c}=\left[\begin{array}{cccc}
0 & 1 & 0 & 0 \\
-\frac{k_{o}+\sum_{i=1}^{6} i k_{i a} \delta_{a}^{i-1}}{m_{a}} & -\frac{d_{o}+\sum_{i=0}^{4} d_{i a} \delta_{a}^{i}}{m_{a}} & \frac{k_{o}}{m_{a}} & \frac{d_{o}}{m_{a}} \\
0 & 0 & 0 & 1 \\
\frac{k_{o}}{m_{b}} & \frac{d_{o}}{m_{b}} & -\frac{k_{o}+k_{b}}{m_{b}} & -\frac{d_{o}+d_{b}}{m_{b}}
\end{array}\right] \\
& B_{c}=\left[\begin{array}{llll}
0 & \frac{N_{a} \varepsilon h_{z}}{2 m_{a} g D_{a}} & 0 & 0
\end{array}\right]^{T} C_{c}=\left[\begin{array}{lll}
k_{o} & d_{o}-k_{o} & -d_{o}
\end{array}\right] \\
& X_{c}=\left[\begin{array}{llll}
\tilde{y}_{a} & \dot{\tilde{y}}_{a} & \tilde{y}_{b} & \dot{\tilde{y}}_{b}
\end{array}\right]^{T}
\end{aligned}
$$

where $A_{c} \in \mathbb{R}^{n \times n}, B_{c} \in \mathbb{R}^{n \times m}$ and $C_{c} \in \mathbb{R}^{p \times n}$, with $n=4, m=1$ and $p=1 . \Delta_{c}^{\prime}=\left[\begin{array}{ll}k_{o} & d_{o}\end{array}\right]$ and $\Delta_{c}\left(\Delta_{c}^{\prime}\right)=\left[\begin{array}{ll}\Delta_{c}^{\prime} & d_{\text {sphere }}\end{array}\right] . \quad \tilde{y}_{a}$ is the variation of $y_{a}$ around the operating point $\delta_{a}$. For sake of readability, $\Delta_{c}\left(\Delta_{c}^{\prime}\right)$ is denoted as $\Delta_{c}$.

A set of 65 Linear Time Invariant (LTI) models are derived from eq. (5). Stiffness $k_{o}$, damping $d_{o}$ and diameter $d_{\text {sphere }}$ are matched for each object in the dataset. Bode diagrams of the elementary LTI models obtained from eq. (5) are shown in Fig. 6. The values of $\delta_{a}$ in Fig. 6 can be deduced from Fig. 3 considering that $\delta_{a}=100 \mu \mathrm{m}-$ $d_{\text {sphere }}$.

The force sensor provides its output with a delay $T_{r}=$ $0.3 \mathrm{~ms}$. This delay has to be taken into account for the controller synthesis. As such, $F_{c d}$ refers to the delayed gripping force signal. The delay is added to the model of eq. (5) by means of a first order Padé approximation as follows:

$$
G_{\text {delay }}=\frac{F_{c d}}{F_{c}}=e^{-T r s} \simeq \frac{1-\frac{T_{r}}{2} s}{1+\frac{T_{r}}{2} s}
$$

The order of the state space model is therefore increased by 1 . Considering $\left(A_{\text {delay }}, B_{\text {delay }}, C_{\text {delay }}, D_{\text {delay }}\right)$ the state space representation of $G_{\text {delay }}$, the uncertain LTI model becomes:

$$
\begin{gathered}
G_{c d}\left(\Delta_{c}\right)\left\{\begin{array}{l}
\dot{X}_{c d}=A_{c d}\left(\Delta_{c}\right) X_{c d}+B_{c d} U \\
F_{c d}=C_{c d}\left(\Delta_{c}^{\prime}\right) X_{c d}
\end{array}\right. \\
A_{c d}\left(\Delta_{c}\right)=\left[\begin{array}{cc}
A_{c}\left(\Delta_{c}\right) & 0_{n \times 1} \\
B_{\text {delay }} C_{c}\left(\Delta_{c}^{\prime}\right) & A_{\text {delay }}
\end{array}\right], B_{c d}=\left[\begin{array}{l}
B_{c} \\
0_{1 \times m}
\end{array}\right] \\
C_{c d}\left(\Delta_{c}^{\prime}\right)=\left[\begin{array}{ll}
D_{\text {delay }} C_{c}\left(\Delta_{c}^{\prime}\right) & C_{\text {delay }}
\end{array}\right] \\
X_{c d}=\left[\begin{array}{ll}
X_{c}^{T} & X_{d}^{T}
\end{array}\right]^{T}
\end{gathered}
$$

where $X_{d}$ is the state of the first order Padé approximation $G_{\text {delay }}$.

Note that a first order Padé approximation may induce additional damping around the resonance. Fig. 7 and Fig. 8 depict respectively the normalized step responses and the Power Spectral Density (PSD) of the step responses of the uncertain LTI model for three microspheres with (model (7)) and without (model (5)) the delay expressed through Padé approximation. Based on the obtained results, the Padé approximation for $T_{r}=0.3 \mathrm{~ms}$ do not have a significant effect on the damping around the resonance.

\section{ROBUST CONTROL DESIGN}

Control specifications are defined in terms of precision, closed loop bandwidth and damping as required in a micromanipulation task. For nominal performance, control specifications are given as: (i) The closed loop response time of the system must be lower than $15 \mathrm{~ms}$. (ii) No overshoot is admitted. An overshoot of a few $\mu N$ can damage the manipulated object. (iii) The maximum static error must be lower than $0.1 \%$.

The proposed control strategy is based on an output feedback eigenstructure assignment (ESA). The method uses an equivalent Luenberger observer formulation and a multimodel design to satisfy robustness with respect to parametric uncertainties. The purpose of the observer structure is to estimate a linear relation of the states and not an exact observation of the plant states as performed for instance with the Kalman filter.

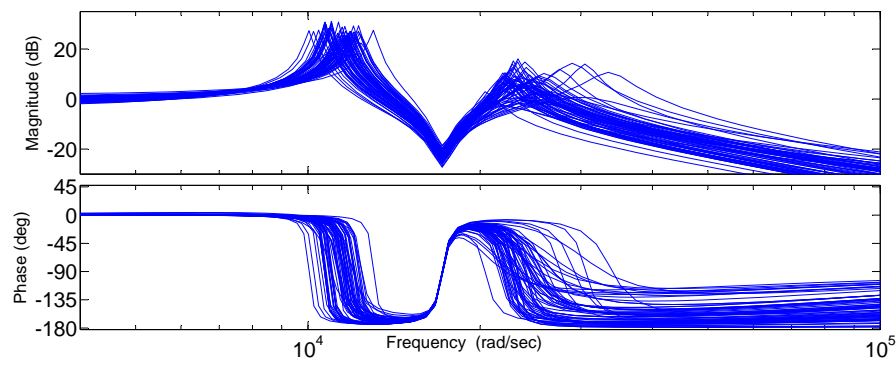

Fig. 6. Bode diagrams of the set of LTI models. 


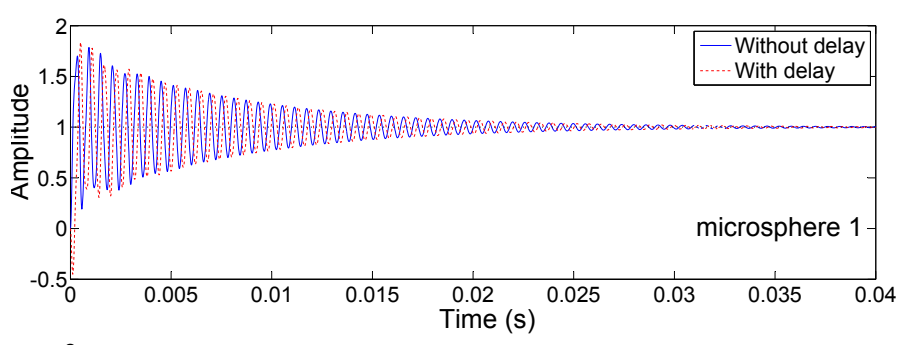

Lemma 1: Given an eigenvalue $\lambda_{i}\left(\Delta_{c 1}\right)$, the triplet $\Gamma=$ $\left(\lambda_{i}\left(\Delta_{c 1}\right), v_{i}\left(\Delta_{c 1}\right), \omega_{i}\left(\Delta_{c 1}\right)\right)$ satisfying

$$
\left[A_{c d}\left(\Delta_{c 1}\right)-\lambda_{i}\left(\Delta_{c 1}\right) I_{n+1}-B_{c d}\right]\left[\begin{array}{l}
v_{i}\left(\Delta_{c 1}\right) \\
\omega_{i}\left(\Delta_{c 1}\right)
\end{array}\right]=0
$$

is assigned by the static gain $K_{c}$ if and only if

$$
K_{c} C_{c d}\left(\Delta_{c 1}^{\prime}\right) v_{i}\left(\Delta_{c 1}\right)=\omega_{i}\left(\Delta_{c 1}\right)
$$

where $v_{i}\left(\Delta_{c 1}\right)$ and $\omega_{i}\left(\Delta_{c 1}\right)$ are respectively the eigenvector and the input direction of the closed loop system.

This control strategy has two main limitations: (i) the degrees of freedom of the controller (i.e. number of triplets that can be assigned in closed loop) is limited by the number of outputs $p$ of the system, (ii) the controller is generally not robust against the variation of uncertain and non-linear parameters.

The latter issue is addressed in the next section using a multimodel eigenstructure assignment approach. For the former, to offer additional degrees of freedom required for the simultaneous resolution of linear constraints of eq. (9) where eigenvectors $v_{i}\left(\Delta_{c 1}\right)\left(1<i<r_{1}\right.$ with $r_{1}=$ the number of multimodel constraints) are distinct, it is necessary to increase the number of outputs.

As such, to assign $r_{1}$ eigenvalues to the fixed parameter system of eq. (7), a set of $n_{c}\left(n_{c}=r_{1}-p\right)$ observers are added.

Lemma 2: The system defined by (see Fig. 9)

$$
\frac{d z_{i}}{d t}=\pi_{i} z_{i}-t_{\pi_{i}} F_{c d}+u_{\pi_{i}} B_{c d} U
$$

where:

$$
u_{\pi_{i}} A_{c d}\left(\Delta_{c 1}\right)+t_{\pi_{i}} C_{c d}\left(\Delta_{c 1}^{\prime}\right)=\pi_{i} u_{\pi_{i}}
$$

with $u_{\pi_{i}}, t_{\pi_{i}}$ and $\pi_{i}$ are the parameters of the observer with appropriate dimensions

is an observer of the variable $z_{i}=u_{\pi_{i}} X_{c d}$ and the observation error $\varepsilon_{i}=z_{i}-u_{\pi_{i}} X_{c d}$ satisfies $\frac{\partial \varepsilon_{i}}{\partial t}=\pi_{i} \varepsilon_{i}$.

This Lemma implies that a linear relation of the states $u_{\pi_{i}} X_{c d}$ can be estimated by an observer. This increases the number of outputs and offers additional degrees of freedom to the controller. It then allows assigning as many additional triplets as the number of observations. Such property is essential to consider multimodel eigenstructure assignment. Furthermore, let's note that in the case of multimodel assignment each additional elementary observer is

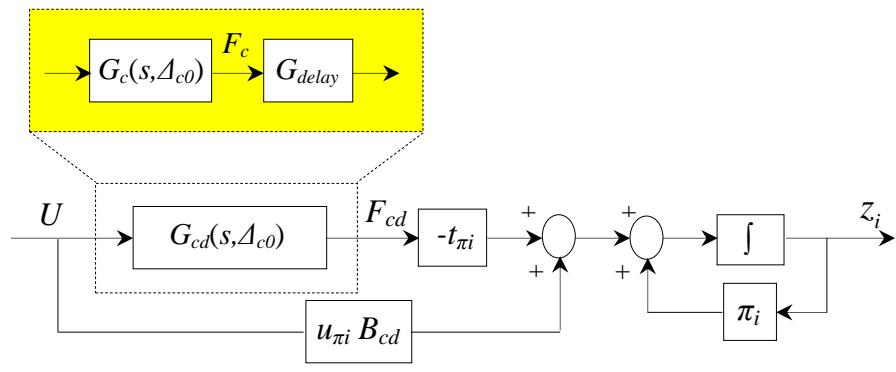

Fig. 9. Elementary observer of $z_{i}=u_{\pi_{i}} X_{c d}$. 


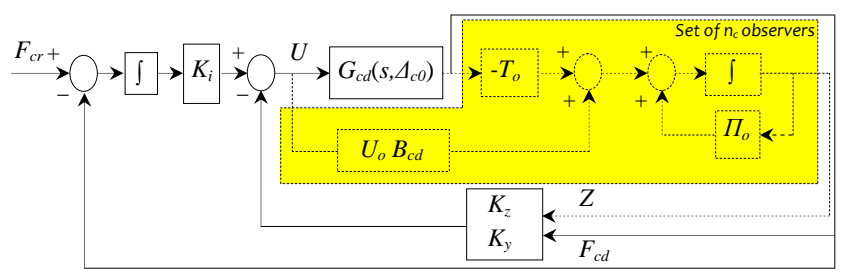

Fig. 10. Observer based control scheme.

designed with respect to the current system configuration (on which the separation principle is guaranteed)

For $n_{c}$ observers, the following notations are introduced:

$$
U_{o} A_{c d}\left(\Delta_{c 1}\right)+T_{o} C_{c d}\left(\Delta_{c 1}^{\prime}\right)=\Pi_{o} U_{o}
$$

with :

$$
\begin{gathered}
U_{o}=\left[\begin{array}{l}
u_{\pi 1} \\
\vdots \\
u_{\pi_{n c}}
\end{array}\right] T_{o}=\left[\begin{array}{l}
t_{\pi 1} \\
\vdots \\
t_{\pi_{n c}}
\end{array}\right] \prod_{o}=\left[\begin{array}{ccc}
\pi 1 & \cdots & 0 \\
\vdots & \ddots & \vdots \\
0 & \cdots & \pi_{n c}
\end{array}\right] \\
Z=\left[\begin{array}{lll}
z_{1} & \ldots & z_{n c}
\end{array}\right]^{T}
\end{gathered}
$$

where $U_{o} \in \mathbb{C}^{n_{c} \times(n+1)}, T_{o} \in \mathbb{C}^{n_{c} \times p}$ and $\Pi_{o} \in \mathbb{C}^{n_{c} \times n_{c}}$.

Therefore, the control problem consists now in finding a gain matrix $K_{c}=\left[\begin{array}{ll}K_{y} & K_{z}\end{array}\right]$ such that the system

$$
\left\{\begin{array}{l}
\dot{X}_{c d}=A_{c d}\left(\Delta_{c 1}\right) X_{c d}+B_{c d} U \\
\frac{d Z}{d t}=\Pi_{o} Z-T_{o} F_{c d}+U_{o} B_{c d} U \\
F_{c d}=C_{c d}\left(\Delta_{c 1}^{\prime}\right) X_{c d}
\end{array}\right.
$$

controlled by the input

$$
U=-K_{y} F_{c d}-K_{z} Z
$$

has the expected performance.

The problem remains now to assign the closed loop eigenstructure of the system:

$$
\left\{\begin{array}{l}
\dot{X}_{c d}=A_{c d}\left(\Delta_{c 1}\right) X_{c d}+B_{c d} U \\
{\left[\begin{array}{l}
F_{c d} \\
Z
\end{array}\right]=\left[\begin{array}{l}
C_{c d}\left(\Delta_{c 1}^{\prime}\right) \\
U_{o}
\end{array}\right] X_{c d}}
\end{array}\right.
$$

by using the augmented static feedback $K_{c}=\left[K_{y} K_{z}\right]$.

From the separation principle, the resulting eigenstructure of the closed loop system (15) is made up of the assigned closed loop poles plus the open loop observer dynamics (i.e. $\Pi_{o}$ ). One of the main characteristics of the control strategy is that the eigenstructure of the observer has not effect on the eigenstructure of the closed loop system which is advantageous in the multimodel design.

Moreover, in order to satisfy the closed loop precision specification, this model is augmented by an integrator. The control law as depicted in Fig. 10 is now expressed as:

$$
U=K_{i} \int\left(F_{c r}-F_{c d}\right)-K_{y} F_{c d}-K_{z} Z
$$

where $K_{i}$ is the gain of the integrator. The controller's gain is expressed as $K_{c}=\left[\begin{array}{lll}K_{y} & K_{z} & K_{i}\end{array}\right]$

The order of the open loop system now equals 5 and $p+1+n_{c}$ measurements are hence available: the output of the system $F_{c d}$, the signal $F_{c r}-F_{c d}$ and the outputs of the observer $Z$.

\subsection{ESA with variable $\Delta_{c}$}

The output feedback controller satisfies the required closed loop performance only for $\Delta_{c}=\Delta_{c 1}$. Hence, robustness is no longer guaranteed for the entire set of LTI models. Let us now assume that in the set of $q$ LTI models, $r_{i}$ triplets have to be assigned for each model $G_{c d}\left(\Delta_{c i}\right)$. Therefore $\sum_{i=1}^{q} r_{i}$ linear constraints of the form:

$$
\begin{gathered}
\left(A_{c d}\left(\Delta_{c 1}\right)-\lambda_{1}\left(\Delta_{c 1}\right) I_{n+1}\right) v_{1}\left(\Delta_{c 1}\right)-B_{c d} \omega_{1}\left(\Delta_{c 1}\right)=0 \\
\vdots \\
\left(A_{c d}\left(\Delta_{c q}\right)-\lambda_{r_{q}}\left(\Delta_{c q}\right) I_{n+1}\right) \\
v_{r_{q}}\left(\Delta_{c q}\right)-B_{c d} \omega_{r_{q}}\left(\Delta_{c q}\right)=0
\end{gathered}
$$

have to be satisfied.

Multimodel constraints are therefore defined by :

$$
\begin{gathered}
K_{c} C_{c d}\left(\Delta_{c 1}^{\prime}\right) v_{1}\left(\Delta_{c 1}\right)=\omega_{1}\left(\Delta_{c 1}\right) \\
\vdots \\
K_{c} C_{c d}\left(\Delta_{c q}^{\prime}\right) v_{r_{q}}\left(\Delta_{c q}\right)=\omega_{r_{q}}\left(\Delta_{c q}\right)
\end{gathered}
$$

The output feedback gain $K_{c}$ must be defined to satisfy this set of multimodel constrains. If the $q$ LTI models correspond to worst-case models, the multimodel synthesis leads to an output feedback controller that meets the robustness performance and the closed loop stability in this set. A solution to satisfy eq. (18) is to schedule the static gain $K_{c}$ by an interpolation formula and to use $\Delta_{c i}$ as the scheduling variable. Let's note that here uncertain parameters, namely the stiffness, the damping and the diameter of the microspheres, are not directly accessible. Yet, it is important to notice that elementary observers are designed step by step during the multimodel procedure by insuring the separation principle relatively to the considered configuration of the system. It is done through Proposition 1 (Magni et al. [1999])

Proposition 1: Given $U_{o} \in \mathbb{C}^{n_{c} \times(n+1)}, T_{o} \in \mathbb{C}^{n_{c} \times p}$ and $\Pi_{o} \in \mathbb{C}^{n_{c} \times n_{c}}$ satisfying eq. (12) such that the interconnection between $\left\{\pi_{1}, \ldots, \pi_{n c}\right\}$ and $\left\{\lambda_{1}\left(\Delta_{c 1}\right) \ldots\right.$, $\left.\lambda_{r_{1}}\left(\Delta_{c 1}\right), \ldots, \lambda_{1}\left(\Delta_{c q}\right), \ldots, \lambda_{r_{q}}\left(\Delta_{c q}\right)\right\}$ is an empty set. For each assignment, a vector $\gamma_{j}\left(\Delta_{c i}\right) \quad(1<\mathrm{j}<$ number of assigned eigenstructures) related to a given LTI model $G_{c d}\left(\Delta_{c i}\right)$ is defined by eq. (19). The dynamic controller satisfies the set of equations (18) if and only if eq. (20) is satisfied where $K_{c}=\left[\begin{array}{lll}K_{y} & K_{z} & K_{i}\end{array}\right]$.

In order to define the multimodel constraints, an iterative procedure is performed as follows:

Step 1: Initialization Design of an output feedback controller (through Proposition 1) with a set of observers on a nominal model $G_{c d}\left(\Delta_{c 1}\right)$. At this step, any fixed value of $\Delta_{c}=\Delta_{c 1}$ can be used from the set of the 65 LTI models. The gain $K_{c}$ is designed considering the following multimodel constraints:

$$
\begin{gathered}
K_{c} C_{c d}\left(\Delta_{c 1}^{\prime}\right) v_{1}\left(\Delta_{c 1}\right)=\omega_{1}\left(\Delta_{c 1}\right) \\
\vdots \\
K_{c} C_{c d}\left(\Delta_{c 1}^{\prime}\right) v_{r_{1}}\left(\Delta_{c 1}\right)=\omega_{r_{1}}\left(\Delta_{c 1}\right)
\end{gathered}
$$




$$
\begin{aligned}
& \gamma_{j}\left(\Delta_{c i}\right)=\left(\lambda_{j}\left(\Delta_{c i}\right)-\Pi_{o}\right)^{-1}\left(U_{o} B_{c d} \omega_{j}\left(\Delta_{c i}\right)-T_{o} C_{c d}\left(\Delta_{c i}^{\prime}\right) v_{j}\left(\Delta_{c i}\right)\right) \\
& {\left[\begin{array}{lll}
K z & K y & K i
\end{array}\right]=\left[\begin{array}{lll}
\omega_{1}\left(\Delta_{c 1}\right) & \cdots & \omega_{r_{q}}\left(\Delta_{c q}\right)
\end{array}\right]\left[\begin{array}{ccc}
\gamma_{1}\left(\Delta_{c 1}\right) & \cdots & \gamma_{r_{q}}\left(\Delta_{c q}\right) \\
C_{c d}\left(\Delta_{c 1}^{\prime}\right) v_{1}\left(\Delta_{c 1}\right) & \cdots & C_{c d}\left(\Delta_{c q}^{\prime}\right) v_{r_{q}}\left(\Delta_{c q}\right)
\end{array}\right]^{-1}}
\end{aligned}
$$

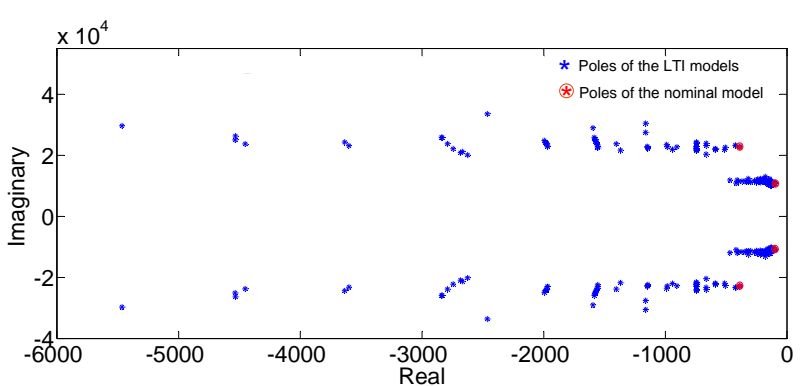

Fig. 11. Pole map of the open loop uncertain LTI model $G_{c d}\left(\Delta_{c}\right)$.

Step 2: Analysis Perform a worst-case analysis (e.g. pole map or $\mu$-synthesis) for a finite number of closed loop LTI models in the parametric space (65 LTI closed loop models). Stop, if the synthesis meets control specifications for all the selected LTI models. Else, identify a worst-case model $G_{c d}\left(\Delta_{c(1+i)}\right)$ and continue to Step 3 .

Step 3: Multimodel synthesis Improve the behavior of the LTI model identified in Step 2 respecting the specifications while preserving the closed loop properties of all the models treated before: the gain $K_{c}$ is designed considering the multimodel constraints of the previous step and those corresponding to $G_{c d}\left(\Delta_{c(1+i)}\right)$ :

$$
\begin{gathered}
K_{c} C_{c d}\left(\Delta_{c(1+i)}^{\prime}\right) v_{1}\left(\Delta_{c(1+i)}\right)=\omega_{1}\left(\Delta_{c(1+i)}\right) \\
\vdots \\
K_{c} C_{c d}\left(\Delta_{c(1+i)}^{\prime}\right) v_{r_{(1+i)}}\left(\Delta_{c(1+i)}\right)=\omega_{r_{(1+i)}}\left(\Delta_{c(1+i)}\right)
\end{gathered}
$$

This iterative procedure leads to the matrices $K_{y}, K_{z}$, $K_{i}$ required to satisfy robust performance and a stability when $\Delta_{c}$ deviates from the nominal model.

\section{SIMULATION RESULTS AND ANALYSIS}

The uncertain LTI model of eq. (7) is used to define a set of 65 LTI models corresponding to the number of characterized microspheres. The pole map of the open loop system is shown in Fig. 11. From the set of the 65 LTI models, the nominal model is selected such that its dominant pole has the highest real value (i.e. LTI model with the longest response time). This model is called $G_{c d}\left(\Delta_{c 1}\right)$. It corresponds to the $8^{t h}$ microsphere of the model 3 .

\subsection{Simulation}

As defined in eq. (12), the triplet $\left\{U_{o}, T_{o}, \Pi_{o}\right\}$ of the observer is related to the matrices $A_{c d}\left(\Delta_{c 1}\right)$ and $C_{c d}\left(\Delta_{c 1}^{\prime}\right)$ of a nominal model $G_{c d}\left(\Delta_{c 1}\right)$. Therefore, when the system deviates from the nominal conditions $\left(\Delta_{c}\right.$ is different from $\Delta_{c 1}$ ) the relation (12) is no longer valid. As a consequence, during Step 3 (Multimodel synthesis), the eigenstructures of the worst case model $G_{c d}\left(\Delta_{c(1+i)}\right)$ can not be assigned exactly to the desired positions. In order to precisely assign the eigenstructures of the worst case model $G_{c d}\left(\Delta_{c(1+i)}\right)$ while preserving the closed loop eigenstructures of the models treated before, we propose here to compute a selfscheduled form of the observer.

The order of the controller is equal to the number of observers. In order to obtain a low order controller, only dominant poles of the treated LTI models $G_{c d}\left(\Delta_{c i}\right)$ will be assigned at each step.

Initialization: The $E S A$ with the observer is applied on the nominal model $G_{c d}\left(\Delta_{c 1}\right)$. The number of assigned poles are $r_{1}=4$ (Table 2 ). Therefore, two observers are designed considering lemma 2 . The observer is defined as:

$$
U_{o}=T_{o} C_{c d}\left(\Delta_{c 1}^{\prime}\right)\left(\Pi_{o} I-A_{c d}\left(\Delta_{c 1}\right)\right)^{-1}
$$

where $T_{o}=\left[\begin{array}{ll}1 & 1\end{array}\right]^{T}$ and $\Pi_{o}=10^{4} \times \operatorname{diag}(-2.75,-1.75)$.

The parameter $\Pi_{o}$ is related to the response time of the observer. It is defined such that it is smaller than the eigenvalue assigned by the output $Z$.

The gain $K_{c}=\left[\begin{array}{lll}K_{y} & K_{z} & K_{i}\end{array}\right]$ is computed considering eq. (21). The desired closed loop eigenvalues are: -300 , $-500,-2 \times 10^{3}$ and $-3 \times 10^{3}$.

Analysis: The worst case analysis (see the pole map in Fig. 12.a) shows that stability is not satisfied in the entire set. The pole map shows that a single step (initial computation) is not sufficient to satisfy robust performance and stability and demonstrates the need of a multimodel assignment (Step 3). The worst case model $G_{c d}\left(\Delta_{c 2}\right)$ is defined such that its closed loop poles have the highest real value. It corresponds to the $11^{\text {th }}$ ball of the model 1 .
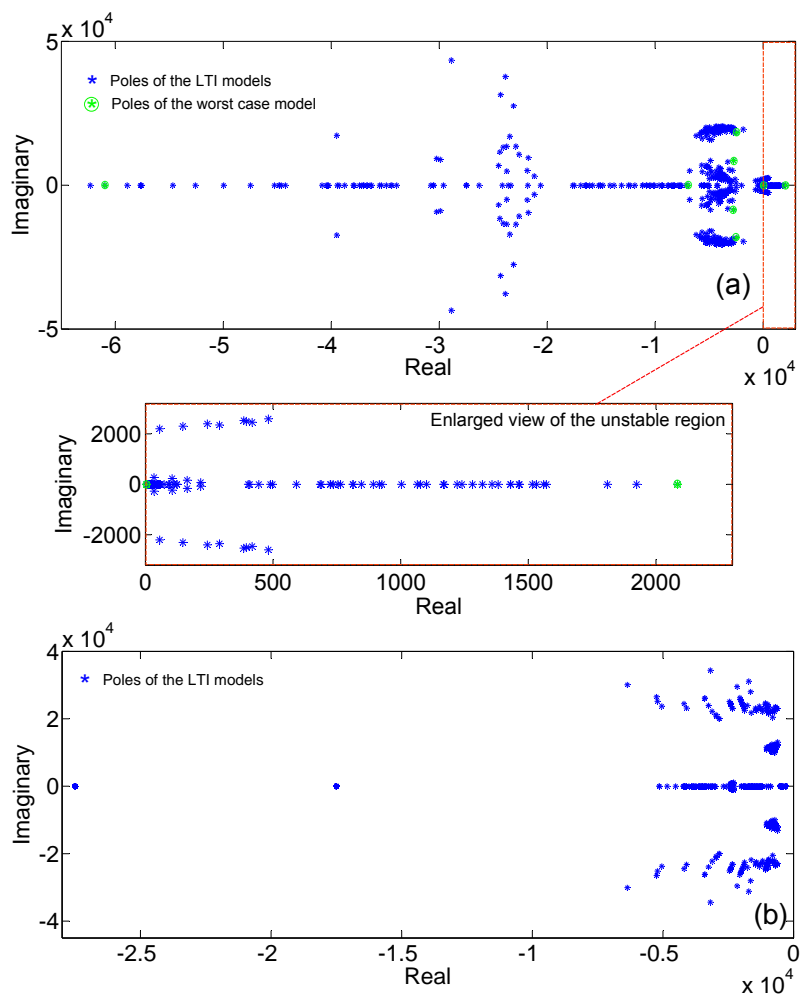

Fig. 12. Pole map obtained during the first step (a) and the third step (b). 

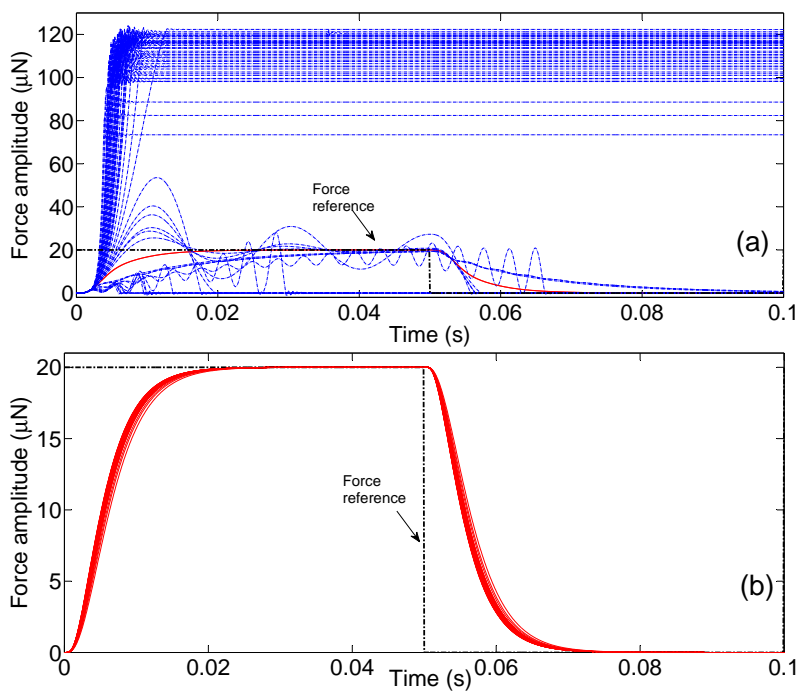

Fig. 13. Step responses $(20 \mu \mathrm{N}$ gripping force reference) of the controlled microgripper when gripping 65 different expancel microspheres: results from Step 1 (a) and Step 3 (b). The step responses satisfying control specifications are in solid line.

Multimodel synthesis: the ESA with the observer is applied to the LTI models $G_{c d}\left(\Delta_{c 1}\right)$ and $G_{c d}\left(\Delta_{c 2}\right)$. The number of assigned poles are $r_{1}=4$ and $r_{2}=2$ (Table 2). Therefore, four observers have been designed, as assigning $r_{1}+r_{2}=6$ eigenstructures requires the use of (6-2) observers. The observer is defined such that:

$$
U_{o}=\left[\begin{array}{c}
U_{o}\left(\Delta_{c 1}\right) \\
U_{o}\left(\Delta_{c 2}\right)
\end{array}\right], T_{o}=\left[\begin{array}{c}
T_{o 1} \\
T_{o 2}
\end{array}\right], \Pi_{o}=\left[\begin{array}{cc}
\Pi_{o 1} & 0_{2 \times 2} \\
0_{2 \times 2} & \Pi_{o 2}
\end{array}\right]
$$

with:

$$
\begin{aligned}
& U_{o}\left(\Delta_{c 1}\right)=T_{o 1} C_{c d}\left(\Delta_{c 1}^{\prime}\right)\left(\Pi_{o 1} I-A_{c d}\left(\Delta_{c 1}\right)\right)^{-1} \\
& U_{o}\left(\Delta_{c 2}\right)=T_{o 2} C_{c d}\left(\Delta_{c 2}^{\prime}\right)\left(\Pi_{o 2} I-A_{c d}\left(\Delta_{c 2}\right)\right)^{-1}
\end{aligned}
$$

where $T_{o 1}=T_{o 2}=\left[\begin{array}{ll}1 & 1\end{array}\right]^{T}$ and $\Pi_{o 1}=\Pi_{o 2}=10^{4} \times$ $\operatorname{diag}(-2.75,-1.75)$.

The gain $K_{c}=\left[K_{y} K_{z} K_{i}\right]$ is computed considering eq. (21) and (22). The desired closed loop eigenvalues are: $-300,-500,-2 \times 10^{3}$ and $-3 \times 10^{3}$ for $G_{c d}\left(\Delta_{c 1}\right)$ and $-300,-400$ for $G_{c d}\left(\Delta_{c 2}\right)$.

Table 2 shows closed loop assigned eigenvalues of the models $G_{c d}\left(\Delta_{c 1}\right)$ and $G_{c d}\left(\Delta_{c 2}\right)$ during Step 1 (analysis) and Step 3 (multimodel synthesis). In Step 3, the eigenvalues of the model $G_{c d}\left(\Delta_{c 2}\right)$ are assigned precisely and the eigenvalues of the model $G_{c d}\left(\Delta_{c 1}\right)$ are the same as the ones assigned in Step 1, preserving the performance of the nominal model.

The worst case analysis (Fig. 12.b) shows that control specifications are satisfied for all the LTI models. The dynamic output feedback controller is of order 4 .

The uncertain LTI model (7) is simulated with the multimodel controller. The force output $F_{c d}$ is used as feedback signal. The reference gripping force $F_{c r}$ is $20 \mu \mathrm{N}$. Fig. 13.a and Fig. 13.b show the controlled gripping force $F_{c d}$ obtained by the multimodel controller designed in Step 1 and Step 3 respectively. A voltage saturation $V_{i n}=140 \mathrm{~V}$ is applied at the input of the open loop model. Instabilities and large errors are clearly visible in Fig. 13.a. These
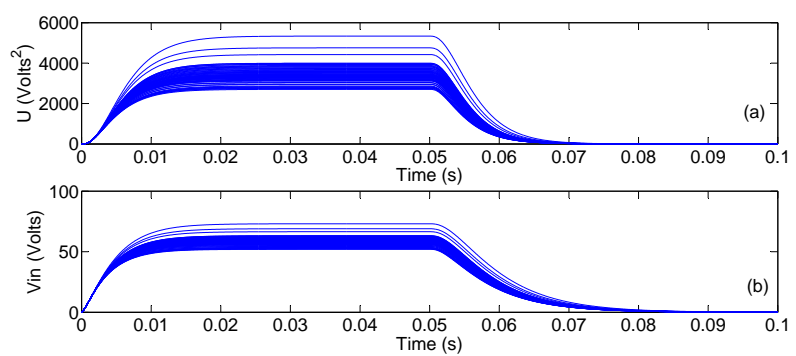

Fig. 14. (a) Control signal $U$ and (b) the square root of the control signal (i.e. $V_{i n}$ ). Results from Step 3 (final controller).

results confirm the worst case analysis and the need for a robust controller. Thanks to the final controller (Fig. 13.b), among the 65 closed loop models, there is no overshoot, no static error and the worst (i.e. longest) response time is $7.9 \mathrm{~ms}$. The control signal $U$ and its square root (i.e. $V_{i n}$ ) obtained in Step 3 (final controller) are shown in Fig. 14.

Table 2. Eigenvalues of $G_{c d}\left(\Delta_{c 1}\right)$ and $G_{c d}\left(\Delta_{c 2}\right)$ controlled by the controllers derived from Step 1 and Step 3. '*' defines the assigned eigenvalues.

\begin{tabular}{lll}
\hline \multirow{2}{*}{ Steps } & $\begin{array}{l}\text { Closed loop eigenvalues } \\
\text { of } G_{c d}\left(\Delta_{c 1}\right)\end{array}$ & $\begin{array}{l}\text { Closed loop eigenvalues } \\
\text { of } G_{c d}\left(\Delta_{c 2}\right)\end{array}$ \\
\hline \hline Step 1 & $-300^{*}$ & $2.085 \times 10^{3}$ \\
& $-500^{*}$ & 4.43 \\
& $-2 \times 10^{3 *}$ & $-2.43 \times 10^{3}+1.82 \times 10^{4} i$ \\
& $-3 \times 10^{3 *}$ & $-2.43 \times 10^{3}-1.82 \times 10^{4} i$ \\
& $-3.75 \times 10^{3}+2.05 \times 10^{4} i$ & $-2.73 \times 10^{3}+8.57 \times 10^{3} i$ \\
& $-3.75 \times 10^{3}-2.05 \times 10^{4} i$ & $-2.73 \times 10^{3}-8.57 \times 10^{3} i$ \\
& $-1.75 \times 10^{4}$ & $-6.96 \times 10^{3}$ \\
& $-2.75 \times 10^{4}$ & $-6.09 \times 10^{4}$ \\
\hline Step 2 & $-300^{*}$ & $-300^{*}$ \\
& $-500^{*}$ & $-400^{*}$ \\
& $-2 \times 10^{3 *}$ & -876.5 \\
& $-3 \times 10^{3 *}$ & $-915.7+1.18 \times 10^{4} i$ \\
& $-628+2.29 \times 10^{4} i$ & $-915.7-1.18 \times 10^{4} i$ \\
& $-628-2.29 \times 10^{4} i$ & $-2.84 \times 10^{3}+2 \times 10^{4} i$ \\
& $-760.6+1.06 \times 10^{4} i$ & $-2.84 \times 10^{3}-2 \times 10^{4} i$ \\
& $-760.6-1.06 \times 10^{4} i$ & $-5.13 \times 10^{3}$ \\
& $-1.75 \times 10^{4}$ & $-1.75 \times 10^{4}$ \\
& $-2.75 \times 10^{4}$ & $-2.75 \times 10^{4}$ \\
\hline
\end{tabular}

\section{EXPERIMENTAL IMPLEMENTATION}

For the real time implementation of the controller, the experimental setup of Fig. 15 is used. It is composed of (a) the microgripper (Fem [2009]), (b) the expancel microspheres, (c) a binocular microscope, (d) a 3 degrees of freedom (dof) positioning system, (e) a controller board (dS [2008]) with a Real Time Interface (RTI), (f) an amplifier for the voltage signal at the output of the controller board and (g) a vibration isolation table. To avoid damaging the gripper, the output of the amplifier is saturated at $140 \mathrm{~V}$.

The controller is designed with the above described procedure through the Matlab/Simulink software.

The controller is then implemented in real time on the microgripper during the grasping with $10 \mathrm{kHz}$ sampling frequency. A desired force reference $F_{c r}$ is applied through the RTI. The measured force signal provided by the capacitive sensor is taken into account in real time by the controller board through an analog/digital interface. The square root of the controller output is applied to the comb drive actuator. 


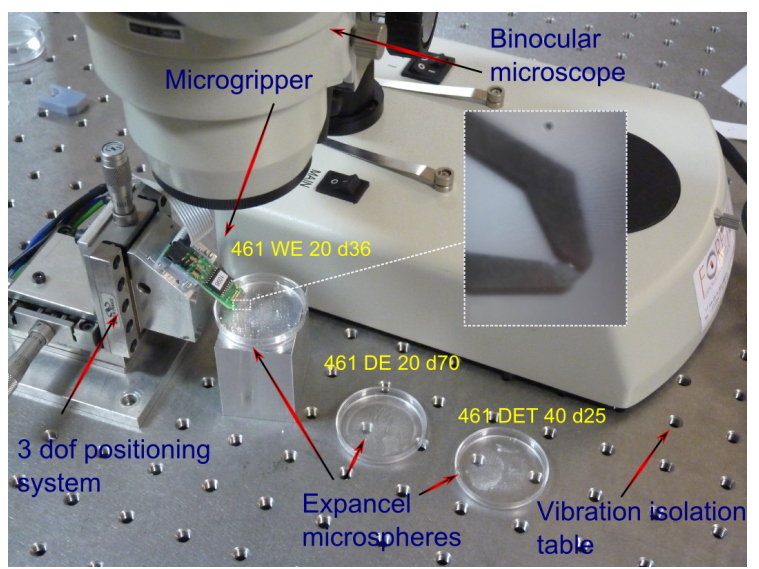

Fig. 15. Experimental setup for microscale gripping force control.

First, the controller designed considering the nominal model (Step 1) is implemented. Five microspheres of each reference (15 microspheres) are then gripped. A reference gripping force of $20 \mu \mathrm{N}$ is applied. As a result (Fig. 16.a), the gripping force saturates ${ }^{1}$ at about $100 \mu \mathrm{N}$ due to instabilities. This excessive force leads to the destruction of some of the gripped spheres.

Afterwards, the multimodel controller (Step 3) is implemented. Five microspheres of each reference (15 microspheres) are gripped. A reference gripping force of $20 \mu \mathrm{N}$ is applied. Fig. 16.b shows that the gripping force reaches the reference force and that control specifications are satisfied. The robust controller is then used to grip a set of 45 microspheres (15 from each reference). As depicted in Fig. 17, all the microspheres are gripped safely and control specifications are satisfied for the entire set.

It is clear from experimental results that the multimodel controller is successful in satisfying robust performance and a robust stability for a safe manipulation of a large number of micrometer sized objects with uncertain characteristics. The control strategy allows obtaining a low order controller which is very advantageous from an application point of view. With traditional $\mathrm{LPV} / \mathrm{H} \infty$ designs, the

1 Recall that a voltage saturation $V_{i n}=140 \mathrm{~V}$ is applied at the input of the comb drive actuator to avoid damaging it.
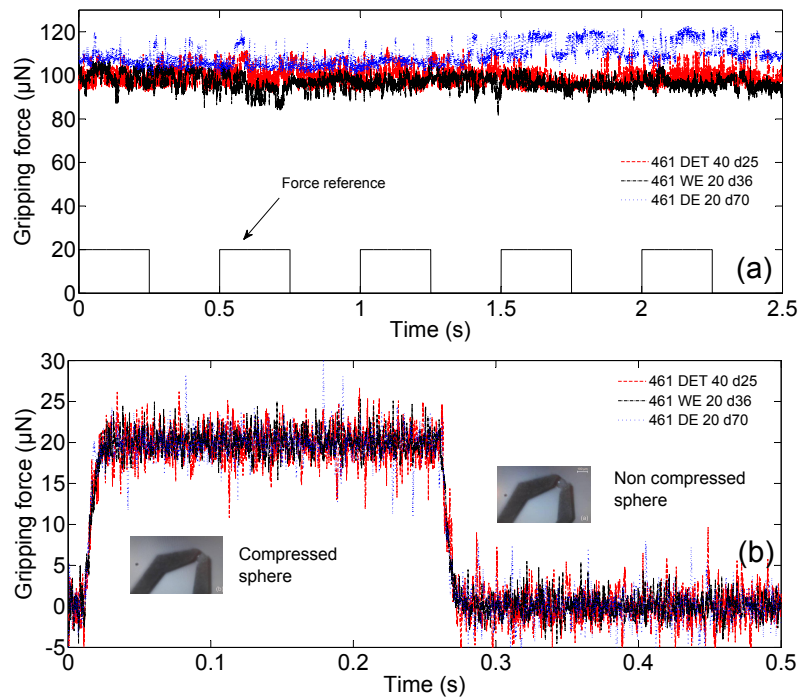

Fig. 16. Gripping force provided by the capacitive sensor for $20 \mu \mathrm{N}$ force reference to 3 different expancel microspheres. The implemented controller is that obtained from the first step (a) and from the third step (b).
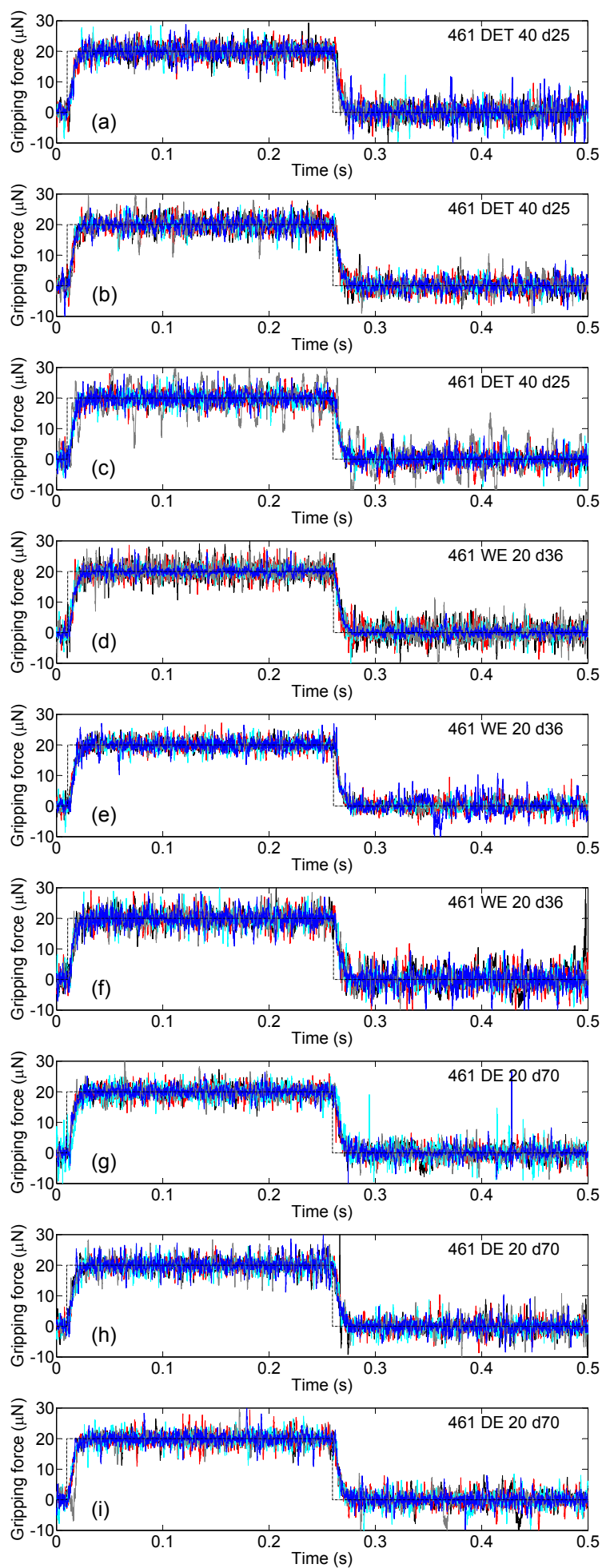

Fig. 17. Gripping force signals provided by the capacitive sensor when applying $20 \mu \mathrm{N}$ force reference to 45 different expancel microspheres (model $3(\mathrm{a}, \mathrm{b}, \mathrm{c})$, model 1 (d,e,f) and model 2 $(\mathrm{g}, \mathrm{h}, \mathrm{i}))$. In each sub figures 5 sub plotted measurements are displayed.

order of the controller is equal to the order of the open loop system + the order of weighting functions. With the coupled microgripper, the order of the controller would be at least equal to 7 (in the case of the use of a single second order weighting function).

\section{APPLICABLE RANGE OF THE CONTROL} DESIGN FOR MICROSCALE OBJECTS GRASPING

The aim of this section is to extend the applicable range of the control design for microscale objects grasping. 
Usually, in the literature, the mechanical properties of spherical objects such as biological cells are evaluated by the characterization of their Young's modulus Radotic et al. [2012]. Nevertheless, very few values of their damping are available.

The controller has been designed taking into account the mechanical properties of the Expancel microspheres whose stiffness can vary from $2.8 \mathrm{~N} / \mathrm{m}$ to $15.7 \mathrm{~N} / \mathrm{m}$. The robustness of the controller can be satisfied for microobjects whose stiffness is in the same range. The relation between the stiffness and the Young's modulus can be defined taking into account a sphere deformation model.

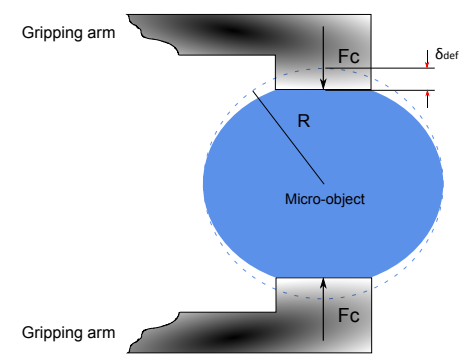

Fig. 18. Schematics of a sphere compressed by gripping arms.

In order to evaluate the applicable range of the control design, a Hertzian deformation model Liu [2009] is used. This model is valid for small deformations (i.e. $\frac{\delta_{\text {def }}}{R}<0.1$ ) Kim et al. [2009] which is the case in this study. The Hertzian model is given as follows:

$$
F_{c}=E \frac{4 \sqrt{R}}{3\left(1-\nu^{2}\right)}\left(\delta_{d e f}\right)^{\frac{3}{2}}
$$

where $E$ is the Young's modulus, $R$ is the radius of the sphere, $\nu$ is Poisson's ratio, $\delta_{\text {def }}$ is the deformation of the sphere and $F_{c}$ is the gripping force (see Fig. 18).

The relation between the Youn's modulus $E$ and the stiffness $k_{o}$ of a sphere is obtained through the calculation of the derivative of $F_{c}$ with respect to $\delta_{d e f}$ around an operating point $\left(F_{c 0}, \delta_{\text {defo }}\right)$. Hence :

$$
E=\frac{3\left(1-\nu^{2}\right)}{4 \sqrt{R}} \frac{2}{3}\left(k_{o}\right)^{\frac{3}{2}} F_{c 0}^{-\frac{1}{2}}
$$

Taking into account the diameter and the stiffness of the studied Expancel microspheres as shown in Fig. 3, a Poisson's ratio $\nu=0.5$ and a gripping force $F_{c 0}=$ $20 \mu \mathrm{N}$ (amplitude of the gripping force reference in the experiments), the Young's modulus of all the studied spheres have been calculated. Results are presented in Fig. 19.

Based on the hypothesis of the Hertzian deformation model and assuming that the spheres are incompressible $(\nu=0.5)$, the controller can satisfy a robust stability during grasping tasks of objects whose Young's modulus ranges from $70.26 \mathrm{kPa}$ to $953.65 \mathrm{kPa}$. For comparison with the Young's modulus of some biological cells, the reader can refer to M. Kemper. [2004], Radotic et al. [2012] and Zdunek et al. [2013].

It is worth noting that the Young's modulus of a spherical object depends on the applied force (i.e. operating point), the diameter of the object and the Poisson's ratio. A very precise and accurate estimation of the Young's modulus of a material requires then a dedicated method

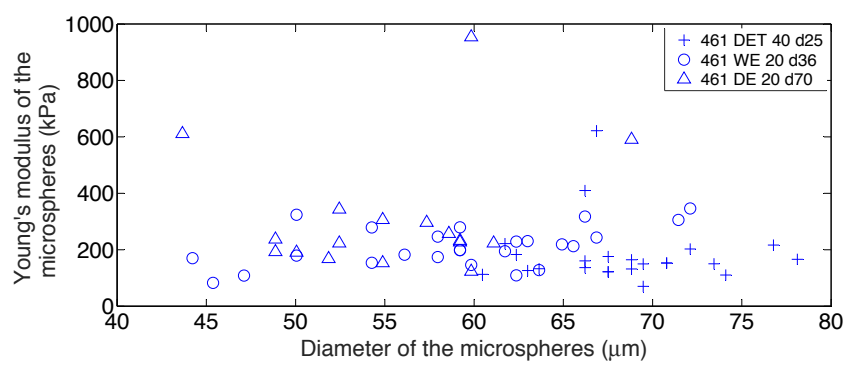

Fig. 19. Disparity map of diameter and Young's modulus of characterized Expancel microspheres.

such as proposed in Radotic et al. [2012] which is not the main purpose of the paper.

\section{CONCLUSION}

This paper has dealt with stability and robustness issues of gripping force control at the microscale. A non-linear electrostatic microgripper with an integrated force sensor has been used to grasp and characterize a set of microspheres. In previous works, a non-linear dynamic model of the actuation mechanism has been proposed. It has been shown experimentally that both the stiffness and the damping of the actuation mechanism are non linear and that they are related to the tip position of the actuated arm. Therefore, during a grasping tasks, the position of the actuated arm in contact with the gripped object is uncertain due to the uncertainty of the object diameter. The latter is considered as the first uncertain parameter in the grasping model. The stiffness and the damping of the manipulated object are the two other uncertain parameters. At the microscale, manipulated objects have enough variation in size, stiffness and damping to induce instabilities during grasping tasks. As such, to satisfy robustness and stability, the controller has been designed taking into account such uncertainties. The methodology proposed in this paper consists first on the experimental identification of the diameter, the stiffness and the damping of a broad set of different microspheres. From an uncertain LTI model, a set of elementary models corresponding to each characterized microsphere are then defined. After that, the gripping force control is designed through a robust observer based controller and a multimodel eigenstructure assignment. The most relevant multimodel constraints required for the synthesis of the robust controller have been defined through a worst case analysis. This analysis allowed the determination that mechanical properties of the $8^{t h}$ microsphere of the model 3 and the $11^{t h}$ microsphere of the model 1 are the most relevant for the determination of the multimodel constraints. The order of the controller is equal to the number of observers, and is potentially low which is of great importance from application point of view. Results show the first experimental demonstration of robust gripping force control at the microscale for the manipulation of a large number of soft materials with varying mechanical properties. The control approach allows the development of new robotic procedures for biological manipulation were samples can vary greatly. Future works will deal with the real time implementation of the controller on a chip embedded within the microgripper to allow grasping strategies that satisfy reliability and safety.

\section{ACKNOWLEDGEMENTS}

This work has been supported by the French national project NanoRobust (ANR 2011 NANO 006).* 


\section{REFERENCES}

Beyeler, F., and Neild, A., and Oberti, S and Bell, D. and Yu, S., and Dual, J., \& Nelson, B. J. (2007). Monolithically fabricated microgripper with integrated force sensor for manipulating microobjects and biological cells aligned in an ultrasonic field. Journal of $M E M S, 16,7-$ 15.

Bolopion, A., Xie, H., Haliyo, S., \& Régnier, S. (2012). Haptic Teleoperation for 3D Microassembly of Spherical Objects. IEEE/ASME T- Mech, 17(1), 116-127.

Boudaoud, M., Le Gorrec, Y., Haddab, Y., \& Lutz, P. (2012). Gain scheduled control strategies for a nonlinear electrostatic microgripper: Design and real time implementation. IEEE CDC, Maui, Hawaii, USA.

Boudaoud M., Haddab, Y., \& Le Gorrec, Y. (2013). Modeling and optimal force control of a nonlinear electrostatic microgripper. IEEE/ASME T- Mech,18, 1130-1139.

Boukallel, M., Girot, M., \& Régnier, S. (2009). Characterization of cellular mechanical behavior at the microscale level by a hybrid force sensing device. $J$ of the mechanical behavior of biomedical materials, 2(3), 297-304.

Carrozza, M. C., and Eisinberg, A., and Menciassi, A., and Campolo, D., and Micera, S., \& Dario, P. (2000). Towards a force-controlled microgripper for assembling biomedical microdevices. J. Micromech Microeng, 10, 271-276.

Chaillet, N., Régnier, S. Microrobotics for Micromanipulation. John Wiley and Sons Inc, ISBN: 978-1-84821186-5, 2010.

Desmaele, D., Boukallel, M., \& Régnier, S. (2012). A resonant structure designed for probing the elastic properties of suspension and adherent cells in liquid environments. J. Micromech Microeng, 22, 115033 (9pp).

Kemper, M. (2004). Development of a Tactile LowCost Microgripper with integrated Force Sensor. International Conference on Control Applications, Taipei, Taiwan.

Kim, K., Liu, X., Zhang, Y., Cheng, J., Yu Wu, X. \& Sun, Y. (2009). Manipulation at the NanoNewton Level: Micrograpsing for Mechanical Characterization of Biomaterials.Intl Conf on Robot and Auto, Kobe, Japan.

Liu, K. K. (2006). Deformation behaviour of soft particles: a review. J. Phys. D: Appl. Phys,39, R189-R199.

Liu, X., Kim, K., Zhang, Y., \& Sun, Y. (2009). Nanonewton force sensing and control in microrobotic cell manipulation. Int J of Robotics Research,28, 1065-1076.

Magni, J. F., Le Gorrec, Y., \& Chiappa, C. (1998). A multimodel-based approach to robust and self-scheduled control design. IEEE CDC, Tampa, FL.

Magni, J. F., Le Gorrec, Y., \& Chiappa, C. (1999). An observer based multimodel control design approach. International Journal of Systems Science, 30, 6168.

Moore, B. C., \& Klein, G. (1976). Eigenvalue selection in the linear regulator combining modal and optimal control. IEEE CDC, Clearwater, Florida, USA.

Muller, D. J., Helenius, J., Alsteens, D., \& Dufrene, Y. F. (2009). Force probing surfaces of living cells to molecular resolution. Nat Chem Biol, 5, 383-390.

Park, J., Kim, S., Kim, D. H., Kim B., Kwon, S. J., Park, J. O., \& Lee, K. I. (2005). Identification and Control of a Sensorized Microgripper for Micromanipulation. IEEE/ASME T- Mech, 10, 601-606.

Poussot-Vassal, C., Sename, O., Dugard, L., Gaspar, P., Szabo, Z., \& Bokor,. J. (2008). A new semi-active suspension control strategy through LPV technique. Control Engineering Practice, 16, 1519-1534.
Radotic, K., Roduit, C., Simonovic, J., Hornitschek, P., Fankhauser, C., Mutavdzic, D., Steinbach, G., Dietler, G., \& Kasas, S. (2012). Atomic Force Microscopy Stiffness Tomography on Living Arabidopsis thaliana Cells Reveals the Mechanical Properties of Surface and Deep Cell-Wall Layers during Growth. Biophysical Journal, 103, 386394.

Rakotondrabe, M., Haddab, Y., \& Lutz, P. (2007). Modelling and $\mathrm{H}$-inf force control of a nonlinear piezoelectric cantilever. IEEE Int Conf on Int Robots and Systems, San Diego, California, USA.

Rakotondrabe, M., \& Le Gorrec, Y. (2010). Force control in piezoelectric microactuators using self scheduled $\mathrm{H} \infty$ technique. Symp on Mech Sys, Cambridge, USA.

Zdunek, A., \& Kurenda,. A. (2013). Determination of the Elastic Properties of Tomato Fruit Cells with an Atomic Force Microscope. Sensors, 13, 12175-12191.

Fem: FT-G100 micropgripper FemtoTools. http://www.femtotools.com, 2009

Akz1: "Product Specification for Expancel Microspheres", microspheres: 461 WE 20 d36 AkzoNobel, Issue 2011.11 (replaces 2010.10)

Akz2: "Product Specification for Expancel Microspheres", microspheres: 461 DE 20 d70 AkzoNobel, Issue 2011.11 (replaces 2010.10)

Akz3: "Product Specification for Expancel Microspheres", microspheres: 461 DET 40 d25 AkzoNobel, Issue 2011.11 (replaces 2010.10)

dS: "DS1103 PPC Controller Board" dSPACE, 2008. 